\title{
QUALIDADE DE VIDA RELACIONADA À SAÚDE DE CRIANÇAS OBESAS
}

Lisiane Schilling Poeta ${ }^{1 *}$, Maria de Fátima da Silva Duarte ${ }^{2}$, Isabela de Carlos Back Giuliano ${ }^{3}$

Trabalho realizado na Universidade Federal de Santa Catarina - UFSC, Florianópolis, SC

*Correspondência:

Rua Dr. Percy Borba, 79

Barreiros

São José - SC

CEP: 88117-035

lisianepoeta@hotmail.com

\section{RESUMO}

OBJEtıvo. Avaliar a qualidade de vida relacionada à saúde de crianças obesas.

Métodos. A pesquisa é do tipo descritiva e de corte transversal. A amostra foi composta por 131 crianças com idades entre 8 e 12 anos, sendo 50 crianças obesas (grupo de estudo) e 81 eutróficas (grupo controle). Todas as crianças participaram da avaliação antropométrica (massa corporal e estatura) para verificação do Índice de Massa Corporal, utilizando os critérios do NCHS-CDC para caracterização do estado nutricional. Para a avaliação da qualidade de vida foi utilizado o questionário PedsQL - versão genérica para crianças, validado para a população brasileira. Foi utilizada a estatística descritiva, o Teste $U$ de Mann Whitney e o teste $t$ de Student não pareado, com nível de significância $p<0,05$. Resultados. O grupo de crianças obesas apresentou qualidade de vida inferior em todos os quatro domínios em relação às crianças eutróficas, com diferença significante nos domínios físico, emocional, social, psicossocial e na qualidade de vida geral. De um total de 100 pontos, a mediana da qualidade de vida geral das crianças obesas foi 69,9 enquanto que das eutróficas foi 82,2.

Conclusão. Na nossa amostra, as crianças obesas apresentaram pior qualidade de vida relacionada à saúde quando comparadas às crianças eutróficas, o que sugere ser um aspecto relevante no planejamento de ações para o controle desta doença.

UnITERMOS: Criança. Obesidade. Qualidade de vida.

\section{INTRODUÇÃO}

A obesidade infantil é um tema de crescente importância devido a sua prevalência e a associação com diversas condições mórbidas. Estudos mostram significativo aumento na prevalência de sobrepeso e obesidade em diversos países e em diferentes faixas etárias, inclusive na população infantil ${ }^{1}$.

A prevalência elevada de obesidade na infância e na adolescência pode resultar no desenvolvimento mais precoce de doenças crônicas na idade adulta, diminuição da qualidade de vida e custo elevado em cuidados de saúde, sendo um fator de risco para doenças como diabetes melito tipo 2 , hipertensão arterial, dislipidemia, síndrome metabólica e eventos clínicos relacionados à aterosclerose, como infarto agudo do miocárdio e acidente vascular encefálico ${ }^{2,3}$.

A obesidade infantil parece também estar envolvida em consequências psicológicas e sociais, pois pode comprometer a auto estima do jovem obeso ${ }^{3}$. Estudos anteriores têm mostrado associação entre baixa qualidade de vida e obesidade. ${ }^{4-6}$

A mensuração da qualidade de vida se tornou um importante indicador de saúde, pois fornece informações a respeito da interferência da condição clínica na vida do paciente, podendo direcionar para políticas públicas que visem à melhoria da qualidade de vida. Esta avaliação deveria ser considerada em crianças com excesso de peso e utilizado como argumento no tratamento ${ }^{4}$. Deveria também servir como um importante parâmetro para avaliação do sucesso do tratamento ${ }^{4}$.

A possibilidade da utilização da avaliação da qualidade de vida na prática clínica tem motivado a realização de estudos e o desenvolvimento de alguns questionários. Para pesquisas no Brasil, foi validado o Questionário de Qualidade de Vida Relacionada à Saúde Pediátrica, "Pediatric Quality of Life Inventory" (PedsQL). ${ }^{7}$ Este foi desenvolvido para ser uma abordagem modular para a aferição da qualidade de vida de crianças, integrando os méritos relativos da abordagem genérica e de doenças específicas $^{8}$. No Brasil, foi realizado um estudo referente à qualidade de vida de jovens obesos, utilizando este questionário como instrumento de medida ${ }^{6}$. Entretanto, no referido estudo, a amostra era constituída por adolescentes matriculados no ensino médio, não envolvendo crianças do ensino fundamental.

A partir do desenvolvimento e validação deste questionário ${ }^{7}$, está sendo possível desenvolver novas pesquisas referentes à qualidade de vida de crianças brasileiras, o que pode ser fonte de subsídios para a elaboração de programas mais eficazes direcionados às crianças obesas. Considerando o exposto, o objetivo deste estudo foi avaliar a qualidade de vida relacionada à saúde (QVRS) de crianças obesas, por intermédio do uso do PedsQL.

1. Aluna do Programa de Pós-Graduação em Educação Física pela Universidade Federal de Santa Catarina - UFSC, Florianópolis, SC

2. Professora titular do Programa de Pós-Graduação em Educação Física pela Universidade Federal de Santa Catarina - UFSC, Florianópolis, SC

3. Professora adjunta do Departamento de Pediatria da Universidade Federal de Santa Catarina - UFSC, Florianópolis, SC 


\section{Métodos}

A amostra total do estudo, observacional e de corte transversal, foi composta por 131 crianças com idades entre 8 e 12 anos, divididas em dois grupos: grupo de estudo (GE) e grupo controle (GC). O GE foi formado por 50 crianças, sendo 23 meninos e 27 meninas, com idade média de 9,76 $\pm 1,28$ anos e com Índice de Massa Corporal (IMC) $\geq$ percentil 95 pelos critérios do NCHS - National Center for Health Statistics ${ }^{9}$. Essas crianças foram recrutadas consecutivamente no ambulatório de cardiologia pediátrica do Hospital Infantil Joana de Gusmão HIJG de Florianópolis/SC - triadas originalmente para um estudo de intervenção interdisciplinar para o tratamento da obesidade, desenvolvido no programa de pós-graduação em Educação Física da Universidade Federal de Santa Catarina, o Estudo PICCOLI (Programa de Intervenção Cardiometabólica em Crianças Obesas Lúdico e Interdisciplinar). O GC foi formado por 81 escolares eutróficos (IMC $<85$ percentil), sendo 35 meninos e 46 meninas, com média de idade de 9,41 $\pm 1,22$ anos provenientes de uma escola pública próxima ao HIJG. Esta escola foi escolhida para controle dos fatores sócio demográficos. Todos os escolares com idades entre 8 e 12 anos foram convidados a participar do GC, totalizando 266 escolares. Porém, foram incluídos no estudo somente os que apresentaram o termo de consentimento assinado pelos pais e apresentavam IMC $<85$ percentil $(\mathrm{n}=$ 81 escolares). Foram excluídos do GE e do GC as crianças com relatos por parte dos pais e/ou professores de dificuldade de aprendizagem, transtorno do déficit de atenção/hiperatividade, deficiência mental e deficiência física. A perda amostral ocorreu por meio da desistência da criança de participar do estudo e/ ou por questionários respondidos de forma incompleta (mais de uma resposta para a mesma questão e/ou questões em branco), ocorrendo em três casos do grupo controle.

Para aferir a massa corporal, foi utilizada uma balança eletrônica, da marca Plena, com precisão de 100 gramas. Para medir a estatura, foi utilizado um estadiômetro portátil, fixado na parede onde não havia rodapé e com escala e precisão de 1 milímetro, tomando-se como pontos de referência o vértex e a região plantar. Foram seguidos os procedimentos descritos por Alvarez e Pavan ${ }^{10}$ durante a avaliação antropométrica. Após essas medidas, foi calculado o IMC pela fórmula: massa corporal (kg) dividida pela estatura $(m)$ elevada ao quadrado.

Foi utilizado um questionário sócio demográfico e clínico, preenchido pelo cuidador da criança, contendo questões subdivididas em itens abertos e fechados, com questões relativas a antecedentes perinatais e pós-natais, antecedentes familiares, rendimento escolar, variáveis relacionadas à atividade física, utilização de medicamentos e problemas de saúde.

Para a avaliação da QVRS foi utilizado o questionário pediátrico de Qualidade de Vida - Pediatric Quality of Life Inventory - PedSQL 4.0 (versão genérica para crianças) ${ }^{8}$, validado para a população brasileira7.

O PedsQL compreende 23 itens divididos em quatro domínios: domínio físico (oito itens); domínio emocional (cinco itens); domínio social (cinco itens); domínio escolar (cinco itens). As questões são respondidas conforme uma graduação de 0 a 4 (nunca, quase nunca, algumas vezes, muitas vezes/frequentemente, quase sempre) e com referência ao último mês vivenciado pela criança. Os itens são aferidos e transformados linearmente para uma escala de 0 a 100
( $0=100 ; 1=75 ; 2=50 ; 3=25$; e 4=0). Realiza-se a soma dos itens e divide-se pelo número de perguntas respondidas. Quanto maior o escore, melhor a qualidade de vida ${ }^{8}$. A qualidade de vida geral é determinada pela média de todos os domínios, enquanto que o aspecto psicossocial é determinado pelo escore médio entre os domínios social, emocional e escolar. O questionário foi idealizado para aferir as dimensões de saúde física, mental e saúde social, seguindo a proposição da Organização Mundial da Saúde, levando-se em consideração também o papel da função escolar. ${ }^{7} \mathrm{O}$ instrumento possui dois formatos paralelos de questionários, um para a criança e outro para os pais, sendo este último desenhado para avaliar a percepção dos pais quanto à QVRS de seus filhos. Neste estudo, foi considerada somente a versão para crianças, pois os questionários encaminhados aos pais das crianças do GC não foram devolvidos em sua maioria (apenas $21 \%$ devolvidos). A autorização do uso do instrumento para fins acadêmicos e não lucrativos foi concedida pelo Instituto Mapi de Pesquisas (Mapi Research Institute).

Para o GE, o PedsQL foi aplicado pela pesquisadora em um ambulatório do HIJG e preenchido pela criança após a explicação de todo questionário (autorrelato), na presença de seu cuidador e da pesquisadora. O questionário sócio demográfico e clínico foi aplicado em forma de entrevista com o responsável pela criança que estava presente no dia da consulta.

As medidas antropométricas do GC foram realizadas em uma sala dentro da própria escola. Para esse grupo, o questionário de qualidade de vida foi aplicado em sala de aula com explicação da pesquisadora e todas as crianças participantes do estudo preencheram os questionários. Entretanto, na análise, foram considerados somente os questionários das crianças com IMC $<$ percentil 85 segundo os critérios do $\mathrm{NCHS}^{9}$ e que não se enquadraram nos critérios de exclusão.

O estudo foi aprovado pelo Comitê de Ética em Pesquisa da Universidade Federal de Santa Catarina - UFSC (protocolo n 302/08).

$\mathrm{Na}$ análise dos dados foi utilizada a estatística descritiva, o teste $U$ de Mann Whitney para dados contínuos não-paramétricos e o teste $t$ de Student não pareado, adotando-se um nível de significância de $p<0,05$.

\section{Resultados}

\section{Dados antropométricos}

Na Tabela 1 são apresentados os dados antropométricos do GE e do GC. O grupo de crianças obesas apresentou valores superiores na estatura, na massa corporal e no IMC em relação ao grupo de crianças eutróficas.

\section{Caracterização do GE}

Em relação à renda familiar mensal, seis relataram renda de até um salário mínimo, 40 de dois a cinco salários e quatro mais de cinco salários mínimos.

A média do peso ao nascer da criança no GE foi de 3.334 gramas, sendo que o peso mínimo foi 1.690 gramas, e o máximo de 4.630 gramas

Dentre as 50 mães questionadas sobre problemas na gestação (GE), uma relatou diabetes e hipertensão arterial, duas relataram obesidade e hipertensão arterial, duas relataram obesidade, diabetes e hipertensão arterial, duas somente diabetes, cinco obesidade, cinco hipertensão e 33 mães não relataram problema algum.

Como antecedente familiar das crianças (pais e avós), a 


\begin{tabular}{|c|c|c|c|}
\hline \multirow{2}{*}{ Antropometria } & Obesas $(n=50)$ & Eutróficas ( $n=81)$ & \multirow[b]{2}{*}{$P$} \\
\hline & $\begin{array}{l}\text { Média } \pm \text { desvio } \\
\text { padrão }\end{array}$ & $\begin{array}{l}\text { Média } \pm \text { desvio } \\
\text { padrão }\end{array}$ & \\
\hline Estatura $(\mathrm{cm})$ & $132,84 \pm 10,00$ & $137,15 \pm 9,81$ & $0,003^{*}$ \\
\hline $\begin{array}{l}\text { Massa corporal } \\
(\mathrm{kg})\end{array}$ & $57,86 \pm 16,44$ & $31,63 \pm 6,81$ & $0,001^{*}$ \\
\hline IMC (kg/m2) & $27,86 \pm 4,77$ & $16,61 \pm 1,61$ & $0,001^{*}$ \\
\hline
\end{tabular}

* Valor de $p<0,05$ diferença significante. Teste t de Student não pareado.

hipertensão arterial foi citada em 26 casos, obesidade em 23 casos, doença cardiovascular em 23 casos, diabetes melito tipo I em 19 casos, diabetes melito tipo II em 13 casos, altos níveis de LDL-colesterol em seis casos, triglicerídeos elevados em cinco casos, diabetes gestacional em quatro casos, baixo HDL-colesterol em três e hiperglicemia em três casos, ocorrendo em todos os casos mais de um problema na família da criança.

Das 50 crianças, nove ingeriam algum tipo de medicamento de forma regular, sendo relatados remédios para hipertensão arterial (cinco casos), ansiedade (dois casos), anti depressivo (um caso) e refluxo (um caso).

Como atividades preferidas durante o lazer, 33 crianças preferiam brincadeiras menos ativas, como por exemplo, assistir à televisão, desenhar, usar o computador, brincar de casinha. Dezessete crianças preferiam brincadeiras mais ativas, como jogar bola, andar de bicicleta, brincar de pega-pega e correr.

Conforme o relato dos cuidadores, o tempo gasto em horas assistindo à televisão ou usando o computador foi em média 3,6 horas por dia, sendo que o tempo mínimo diário foi de uma hora e o máximo foi de oito horas.

Quanto à participação nas aulas de educação física escolar, 41 crianças relataram sempre participar, duas quase sempre participavam, cinco informaram participar somente algumas vezes e duas nunca participavam das aulas.

Qualidade de vida relacionada à saúde

A Tabela 2 apresenta um comparativo entre os escores dos domínios da QVRS entre as crianças obesas e eutróficas. As crianças obesas apresentaram pior qualidade de vida em todos os domínios, com diferença significante nos domínios físico, emocional, social, psicossocial e na qualidade de vida geral.

\section{Discussão}

Este estudo descreve o estado da qualidade de vida relacionada à saúde de escolares obesos, o que pode corroborar a necessidade premente de intervenção nessas crianças, na medida em que agrega à argumentação tradicional a preocupação com aspectos mais subjetivos da vida cotidiana desse grupo, que apresenta um número cada vez mais significativo de indivíduos em todo o país ${ }^{1}$.

Antecedentes familiares de obesidade, hipertensão arterial e diabetes foram encontrados no grupo de crianças obesas. Além disso, problemas durante a gestação também foram relatados, sendo a obesidade gestacional o mais citado. 0 ganho de peso materno durante a gestação tem sido associado ao aumento no risco de obesidade na infância e resistência à insulina ${ }^{11}$. Numa abordagem longitudinal, Boney et al. ${ }^{12}$ demonstraram que os recém nascidos grandes para a idade gestacional e filhos de mães diabéticas ou com obesidade durante a gestação apresentaram maior risco para a síndrome metabólica após os nove anos de idade. Estes achados reforçam argumentos sobre a perpetuação do ciclo da obesidade, da resistência à insulina e de suas consequências a gerações subsequentes, sugerindo a necessidade do controle da obesidade materna quando se planeja combater a obesidade infantil.

Algumas crianças da nossa amostra nasceram com peso acima de $4 \mathrm{~kg}$, sendo que o peso máximo relatado foi 4,63 kg. Tais achados concordam com os de Hirschler et al. ${ }^{13}$, que verificaram a associação entre peso ao nascer em crianças e obesidade, sobrepeso e síndrome metabólica aos nove anos de idade em estudantes argentinos. No modelo de regressão logística, baixo peso ao nascer ( $\leq 2,5 \mathrm{~kg}$ ) mostrou-se como um fator de proteção para sobrepeso e obesidade, enquanto que peso $\geq 4 \mathrm{~kg}$ ao nascer foi associado com maior risco de sobrepeso, obesidade e síndrome metabólica ajustado por sexo e idade. Entretanto, outros estudos têm mostrado associação entre baixo peso ao nascer e doença arterial coronariana ${ }^{14}$. Barker et al. ${ }^{14}$ encontraram que baixo peso ao nascer combinado com ganho rápido de peso na infância entre 3 e 11 anos prediz grandes diferenças na incidência cumulativa de doença coronariana, diabetes melito tipo II e hipertensão arterial, evidenciando que ainda não há consenso sobre tal associação.

Todas as crianças desta amostra eram estudantes de escolas públicas, e a maior parte com renda familiar mensal entre dois e cinco salários mínimos, o que dificultou a análise relacionada ao impacto da classe econômica no risco de obesidade. Os achados relacionando o nível sócio econômico e a obesidade infantil não têm sido consistentes na literatura, mas estudos brasileiros tendem a evidenciar uma associação entre sobrepeso e obesidade e melhores

Tabela 2 - Escores dos domínios da qualidade de vida das crianças obesas e eutróficas, estudo PICCOLI, Florianópolis-SC, 2009

\begin{tabular}{lccc}
\hline \multirow{2}{*}{ Domínios qualidade de vida } & Obesas $(n=50)$ & Eutróficas $(n=81)$ & Mediana [IIC] \\
\cline { 2 - 3 } & Mediana [IIC] & $90,62[84,37-96,87]$ & $<0,001^{*}$ \\
\hline Físico & $75,00[59,37-81,25]$ & $70,00[55,00-85,00]$ & $<0,001^{*}$ \\
Emocional & $50,00[40,00-70,00]$ & $90,00[80,00-95,00]$ & $0,005^{*}$ \\
Social & $80,00[70,00-90,00]$ & $80,00[72,50-90,00]$ & 0,171 \\
Escolar & $80,00[63,75-86,25]$ & $80,00[69,16-86,66]$ & $0,001^{*}$ \\
Psicossocial & $70,83[62,91-78,33]$ & $82,18[72,96-88,74]$ & $<0,001^{*}$ \\
QV geral & $69,92[64,64-78,28]$ & &
\end{tabular}

* Valor de $p<0,05$ diferença significante. Teste $U$ de Mann Whitney.

Dados apresentados em mediana e intervalos interquartílicos (IIC). 
condições sócio econômicas familiares. ${ }^{15}$ Em contrapartida, outro estudo indica que a associação da obesidade com doenças crônicas tem se mostrado mais frequente nas áreas carentes ${ }^{16}$, talvez devido à alimentação inadequada e menor acompanhamento médico.

O lazer preferido de 33 crianças do GE desta amostra constituiuse de brincadeiras menos ativas, e o tempo diário destinado à televisão ou ao computador variou entre uma e oito horas. Este é um dado preocupante, uma vez que o estilo de vida sedentária é um dos fatores relacionados ao desenvolvimento da obesidade pediátrica, além de estar implicado em outros agravos, como depressão, baixa auto estima, entre outros ${ }^{3}$. Graf et al. ${ }^{17}$ encontraram relação direta entre o tempo assistindo a televisão e o aumento da adiposidade em escolares, sendo que a incidência de obesidade aumentou com o tempo dedicado à televisão por mais de três a cinco horas diárias. Essa associação talvez possa ser explicada por ser essa uma atividade sedentária, agravada pelo maior estímulo e oportunidade de consumo de alimentos calóricos, e por estarem as crianças mais expostas às propagandas televisivas relacionadas aos produtos alimentícios de alta densidade calórica e de gorduras. Esses resultados sugerem estarem implicados fatores genéticos, ambientais e comportamentais como possíveis fatores de risco para a ocorrência de obesidade, reforçando dados da literatura.

Os achados deste estudo apontam para uma relação significante entre obesidade e baixa QVRS. Crianças obesas apresentaram escores inferiores às crianças eutróficas em todos os domínios da qualidade de vida, sugerindo que o impacto desta condição é global. Os resultados concordam com os achados de outros autores, onde a qualidade de vida de crianças e adolescentes com sobrepeso/obesidade foi inferior em relação aos nãoobesos, segundo relato dos pais e/ou da própria criança ${ }^{4-6,18-21}$.

Ravens-Sieberer et al. ${ }^{4}$ mostraram que crianças obesas apresentaram escores inferiores de qualidade de vida, comparado aos de crianças com asma, nos domínios avaliados pelo questionário alemão KINDL (relato dos pais e das crianças), sendo que as meninas apresentaram escores inferiores aos meninos nos aspectos físico, bem-estar psicológico, auto estima e escore total. A pior qualidade de vida nas meninas pode estar relacionada à maior preocupação com a imagem corporal na comunidade observada, interferindo principalmente nos domínios emocional e social. No presente estudo não foi analisada a qualidade de vida considerando o sexo devido ao tamanho da amostra.

Schwimmer et al. ${ }^{18}$, utilizando o PedsQL, verificaram que crianças e adolescentes obesos apresentaram pior qualidade de vida, em todos os domínios, em relação aos não-obesos, similarmente a crianças com câncer, pelo relato dos pais e das crianças. Este resultado é alarmante, pelas reconhecidas mudanças na vida de crianças portadoras de câncer devido ao tratamento e, consequentemente, dificuldades na manutenção de suas atividades diárias e todo o estresse da criança e da família. Mesmo sem uma exposição tão intensa à intervenção médica sofrida pelas crianças com câncer, as obesas apresentaram qualidade de vida similar àquelas, sugerindo um prejuízo grave destas somente pela sua condição clínica. Na Austrália, Williams et $a^{19}{ }^{19}$., utilizando o PedsQL, encontraram escores significantemente inferiores nos domínios físico e social de crianças com sobrepeso comparadas às eutróficas, pelo relato dos pais e das crianças. Porém, a alteração do aspecto emocional e escolar não apresentou significância entre os grupos, demonstrando menores impactos na QVRS da amostra que o observado em outras amostras clínicas, utilizando o mesmo instrumento de avaliação, estudos estes $^{18,19}$ que reforçam a impressão da presente pesquisa, do impacto negativo da obesidade nos domínios da QVRS.

Recentemente, Tsiros et al. ${ }^{20}$ defenderam a ideia, por meio de uma revisão mais abrangente, que a maioria dos estudos apontam para uma relação inversa entre IMC e qualidade de vida da população pediátrica, com prejuízos consistentes nos domínios físico e social. Utilizando o PedsQL (autorrelato da criança), Tyler et al. ${ }^{21}$ encontraram que jovens severamente obesos apresentaram pior qualidade de vida nos aspectos físico e psicossocial, quando comparados a outros três grupos: peso normal, risco de obesidade e obesidade. Entretanto, no mesmo estudo, somente o domínio psicossocial foi inferior no grupo de jovens com obesidade severa comparado ao grupo de obesos, enquanto que o aspecto físico não diferiu entre esses dois grupos.

Encontrou-se diferença estatisticamente significante entre os grupos GE e GC nos domínios físico, emocional, social, psicossocial e na qualidade de vida geral. Embora no domínio escolar as crianças eutróficas tenham apresentado escores superiores, a diferença entre os grupos não foi significante. Segundo Williams et al. ${ }^{19}$ parece viável que algumas dimensões podem ser mais afetadas nas crianças com excesso de peso, sendo mais evidente nos domínios físico e social, enquanto os aspectos emocional e escolar permanecem relativamente preservados. Entretanto, no presente estudo o aspecto emocional foi o mais afetado dentre os domínios analisados, com diferença significante entre os grupos, como encontrado em outras pesquisas ${ }^{22,23}$. No questionário PedsQL este domínio contempla questões relacionadas a sentimentos de medo, tristeza, raiva, preocupação e dificuldade para dormir. Algumas crianças obesas dessa amostra relataram sentimentos de tristeza com frequência. Isso parece concordar com o senso comum, pois os sintomas depressivos têm sido apontados como variável que influi de maneira significativa na qualidade de vida, quando se trata de jovens obesos, sendo associados à menor aderência nos programas de intervenção para controle do peso, formando-se um ciclo vicioso ${ }^{22}$. Em uma amostra de 166 jovens obesos, aproximadamente $11 \%$ apresentaram critérios significativos de sintomas depressivos ${ }^{21}$. Csabi et al. ${ }^{23}$ estudaram crianças obesas em tratamento ambulatorial e encontraram maior proporção de sintomas depressivos em crianças obesas quando comparadas as de peso normal. Por meio de uma revisão da literatura, Luiz et al. ${ }^{24}$ encontraram uma relação entre obesidade infantil e aspectos psicológicos, tais como depressão, ansiedade e déficits de competência social. Para a compreensão deste aspecto, deve-se considerar que muitas vezes as crianças obesas sofrem estigmatização social, podendo causar impacto negativo na qualidade de vida, interferindo principalmente nos aspectos emocionais e sociais.

Dentre os domínios da qualidade de vida avaliados pelo PedsQL, a maior diferença entre os grupos GE e GC na amostra estudada foi no aspecto físico. Isso pode ser devido à dificuldade relatada pelas crianças em caminhar, correr, praticar atividades esportivas, ou sentirse com pouca energia (algumas das questões do PedsQL referentes ao domínio físico). Determinadas dificuldades podem estar relacionadas a problemas ortopédicos e, consequentemente, alterações na mobilidade. 0 mau alinhamento dos membros inferiores, acompanhado pelo excesso de peso sobre as articulações, pode contribuir no aumento da prevalência de desconforto músculo esquelético em jovens obesos, levando, usualmente, a criança a participar menos de atividades físicas, outro ciclo vicioso evidenciado na fisiopatologia da 
obesidade ${ }^{25}$. Bracco et al. ${ }^{26}$ encontraram que o gasto energético entre as crianças obesas foi maior que o das não obesas por minuto de atividade física realizada, evidenciando um maior esforço despendido pelas crianças obesas para a mesma intensidade de atividade física. Esse esforço maior das obesas pode ser um fator que justificaria o maior tempo de atividade sedentária delas quando comparadas às eutróficas, o que pode ter contribuído para resultados inferiores no domínio físico do PedsQL nos jovens com obesidade.

Os resultados aqui obtidos sugerem que uma proporção substancial de crianças pode estar vivendo com menor QVRS devido à obesidade. Porém, é possível que programas de redução de peso melhorem a qualidade de vida dessas crianças, especialmente no domínio físico ${ }^{27}$.

As crianças obesas deste estudo apresentaram pior qualidade de vida quando comparadas às eutróficas em todos os domínios, com diferença significante no domínio físico, social, emocional, psicossocial e na qualidade de vida geral, o que também deve ser levado em conta no planejamento de ações para o controle desta doença, tanto na preocupação com a adesão ao tratamento quanto no controle também destes aspectos.

É relevante esclarecer que se deve tomar cuidado na generalização destes dados, na medida em que os grupos foram selecionados de uma amostra não representativa da população escolar como um todo. Outra limitação é o fato de não se ter diagnosticado outros problemas de saúde, exceto aspectos cardiovasculares (utilizado para o estudo PICCOLI) que poderiam estar interferindo na qualidade de vida, assim como fatores culturais e sociais, que igualmente poderiam interferir na qualidade de vida e não foram completamente controlados neste estudo, apesar da preocupação de selecionar grupos que vivam próximos à escola e ao ambulatório.

\section{Conflito de interesse: não há}

\section{SUMmarY}

\section{HeALTH-RELATED QUALity OF LIFE OF OBESE CHILDREN}

OBJECTIVE. To evaluate the health-related quality of life of obese children.

Methods. This is a descriptive and cross-sectional study. The sample consisted of 131 children, aged 8 and 12 years, 50 obese children (study group) and 81 with normal weight (control group). All children underwent anthropometric assessment (weight and height) to obtain the body mass index. The criterion of the NCHS-CDC was used to determine nutritional status. Quality of life was assessed by the PedsQL - generic version for children, validated for the Brazilian population. We used descriptive statistics, the $U$ test, the Mann Whitney and unpaired Student $t$ tests, with a significance level of $p<0.05$.

RESULTS. The group of obese children had a lower quality of life in all four areas when compared to normal children, with a significant difference in the physical, emotional, social, psychosocial and overall quality of life. From a total of 100 points, the median of overall quality of life of obese children was 69.9 while that of normal weight was 82.2.

CONCLUSIONS: Obese children had a poorer quality of life related to health when compared to normal children, which should be taken into account when planning for control of obesity. [Rev Assoc Med Bras 2010; 56(2): 168-72]

KEY WORDS: Child. Obesity. Quality of life.

\section{REFERÊNCIAS}

1. Wang Y, Monteiro C, Popkin BM. Trends of obesity and underweight in older children and adolescents in the United States, Brazil, China and trends of obesity and underweight in older children and adolescents in the United States, Brazil, China and Russia. Am J Clin Nutr. 2002;75(6):971-7.

2. World Health Organization (WHO). Preventing and managing the global epidemic. Report of a WHO Consultation. Geneva; WHO; 2000.

3. Abrantes MM, Lamounier JA, Colsimo EA. Prevalência de sobrepeso e obesidade nas regiões sudeste e nordeste. J Pediatr. 2002;78:335-40.

4. Ravens-Sieberer U, Redegeld M, Bullinger M. Quality of life after in-patient rehabilitation in children with obesity. Int J Obes. 2001;25 (Suppl.1):63-5.

5. Friedlander SL, Larkin EK, Rosen CL, Palermo TM, Redline S. Decreased quality of life associated with obesity in school-aged children. Arch Pediatr Adolesc Med. 2003;157:1206-11.

6. Kunkel N, Oliveira WF, Peres MA. Excesso de peso e qualidade de vida relacionada à saúde em adolescentes de Florianópolis, SC. Rev Saúde Pública. 2009·43. 226-35.

7. Klatchoian DA, Len CA, Terreri MTRA, Silva M, Itamoto C, Ciconelli RM, et al. Quality of life of children and adolescents from São Paulo: reliability and validity of the Brazilian version of the Pediatric Quality of Life Inventory TM version 4.0 Generic Core Scales. J Pediatr. 2008;84:308-15.

8. Varni JW, Seid M, Kurtin OS. PedsQL (TM) 4.0: Reliability and validity of the Pediatric Quality of Life Inventory (TM) version 4.0 Generic Core Scales in healthy and patient populations. Med Care. 2001;39:800-12.

9. CDC: Table for calculated body mass index values for selected heights and weights for ages 2 to 20 years. Developed by the National Center for Health Statistics in collaboration with the National Center for Chronic Disease Prevention and Health Promotion; 2000. [cited 2008 aug 20]. Available from: http://www.cdc.gov/growthcharts.

10. Alvarez RB, Pavan AL. Alturas e comprimentos corporais. In: Petroski EL. Antropometria: técnicas e padronizações. $2^{a}$ ed. Santa Maria: Pallotti; 2003.

11. Cho NH, Silverman BL, Rizzo TA, Metzer BE. Correlations between the intrauterine metabolic environment and blood pressure in adolescent offspring of diabetic mothers. J Pediatr. 2000;136:587-92.

12. Boney CM, Verma A, Tucker R, Vohr BR. Metabolic syndrome in childhood: association with birth weight, maternal obesity, and gestational diabetes mellitus. Pediatrics. 2005;115:290-6.

13. Hirschler V, Bugna J, Roque M, Gilligan T, Gonzalez C. Does low birth weight predict obesity/overweight and metabolic syndrome in elementary school children? Arch Med Res. 2008;39:796-802.

14. Barker DJ, Eriksson JG, Forsén T, Osmond C. Fetal origins of adult disease: strength of effects and biological basis. Int J Epidemiol. 2002;31:1235-9.

15. Dutra CL, Araújo CL, Bertoldi AD. Prevalência de sobrepeso em adolescentes: um estudo de base populacional em uma cidade no Sul do Brasil. Cad Saúde Pública. 2006;22:151-62.

16. Alves JB, Galé CR, Souza E, Batty GD. Efeito do exercício físico sobre peso corporal em crianças com excesso de peso: ensaio clínico comunitário randomizado em uma favela no Brasil. Cad Saúde Pública. 2008;24 (suppl 2):353-9.

17. Graf C, Rokarski W, Predel HG, Koch B, Dordel S. Overweight and obesity in childhood - how can physical activity help? Phys Educ Sport. 2006;50:54-9.

18. Schwimmer JB, Burwinkle TM, Varni JW. Health-related quality of life of severely obese children and adolescents. JAMA. 2003;289:1813-9.

19. Williams J, Wake M, Hesketh K, Maher E, Waters E. Health-related quality of life of overweight and obese children. JAMA. 2005;293:70-6.

20. Tsiros MD, Olds T, Buckley JD, Grimshaw P, Brennan L, Walkley J, et al. Health-related quality of life in obese children and adolescents. Int J Obes. 2009;33:387-400.

21. Tyler C, Johnston CA, Fullerton G, Forety JP. Reduced quality of life in very overweight Mexican American adolescents. J Adolesc Health. 2007;40:366-8.

22. Zeller M, Modi A. Predictors of health-related quality of life in obese youth. Obesity. 2006;14:122-30.

23. Csabi G, Tenyi T, Molnar D. Depressive symptoms among obese children. Eat Weight Disord. 2000;5:43-5.

24. Luiz AMG, Gorayeb R, Liberatore Junior RDR, Domingos NAM. Depressão, ansiedade, competência social e problemas comportamentais em crianças obesas. Estud Psicol. 2005;10:371-5.

25. Matsudo SMM, Matsudo VKR. Atividade física e obesidade: prevenção e tratamento. São Paulo: Atheneu; 2008.

26. Bracco MM, Ferreira MBR, Morcillo AM, Colugnati F, Jenovesi JF. Gasto energético entre crianças de escola pública obesas e não obesas. Rev Bras Ciênc Mov. 2002;10:29-35.

27. Fullerton G, Tyler C, Johnston CA, Vincent JP, Harris G, Forety JP. Quality of life in Mexican-American children following a weight management program. Obesity. 2007;15:2553-6.

Artigo recebido: 05/12/09

Aceito para publicação: 22/01/10 